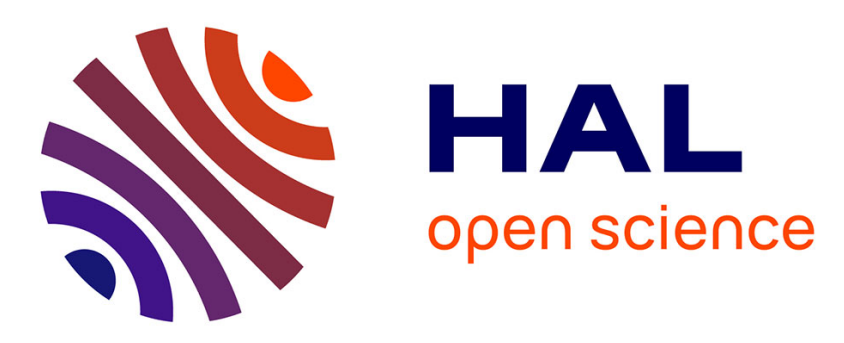

\title{
MODELING A PARALLELISM CONSTRAINT IN ACTIVE CONTOURS. APPLICATION TO THE SEGMENTATION OF EYE VESSELS AND RETINAL LAYERS
}

Itebeddine Ghorbel, Florence Rossant, Isabelle Bloch, Michel Paques

\section{- To cite this version:}

Itebeddine Ghorbel, Florence Rossant, Isabelle Bloch, Michel Paques. MODELING A PARALLELISM CONSTRAINT IN ACTIVE CONTOURS. APPLICATION TO THE SEGMENTATION OF EYE VESSELS AND RETINAL LAYERS. ICIP, Sep 2011, Brussels, Belgium. 10.1109/ICIP.2011.6116546 . hal-02882940

\section{HAL Id: hal-02882940 https://hal.science/hal-02882940}

Submitted on 28 Jun 2020

HAL is a multi-disciplinary open access archive for the deposit and dissemination of scientific research documents, whether they are published or not. The documents may come from teaching and research institutions in France or abroad, or from public or private research centers.
L'archive ouverte pluridisciplinaire HAL, est destinée au dépôt et à la diffusion de documents scientifiques de niveau recherche, publiés ou non, émanant des établissements d'enseignement et de recherche français ou étrangers, des laboratoires publics ou privés. 


\title{
MODELING A PARALLELISM CONSTRAINT IN ACTIVE CONTOURS. APPLICATION TO THE SEGMENTATION OF EYE VESSELS AND RETINAL LAYERS.
}

\author{
Itebeddine GHORBEL ${ }^{1,2,3}$, Florence ROSSANT ${ }^{2}$, Isabelle BLOCH ${ }^{3}$ and Michel PAQUES ${ }^{4}$ \\ ${ }^{1}$ FOVEA Pharmaceuticals Paris, France \\ ${ }^{2}$ ISEP Paris, France \\ ${ }^{3}$ Télécom ParisTech, CNRS LTCI, Paris, France \\ ${ }^{4}$ Clinical Investigation Center 503, Centre Hospitalier National des Quinze-Vingts Paris, France
}

\begin{abstract}
Parametric deformable models are an important technique for image segmentation. In order to improve the robustness of the model, it may be interesting to incorporate a priori information about the shape of the objects to be segmented. In this paper, we propose to add a parallelism constraint. Such a model is relevant in many applications where elongated structures have to be detected. One main advantage of our formulation is that it only needs few parameters to be adjusted in addition to those of traditional snakes. The proposed model has been applied for the segmentation of OCT images of the retina and for the segmentation of retinal vessels. Experimental results, obtained on 25 OCT images and 30 eye fundus images, demonstrated the robustness, flexibility and large potential applicability of this new formulation. The accuracy of the method has been assessed by comparing manual segmentations, made by experts, with the automatic ones.
\end{abstract}

Index Terms - Active contours, Parallelism, Retinal vessel segmentation, Optical Coherence Tomography, Segmentation, Quantitative Evaluation.

\section{INTRODUCTION}

The snake concept, also called "active contour model", was originally introduced by Kass et al. [1] in order to detect the boundary of an object in an image. It consists of a parametric curve $V$, whose evolution is driven by the minimization of an energy function $E$, which includes terms controlling the smoothness of the curve (internal energy $E_{I n t}$ ) and terms related to information image (image energy $E_{\text {Image }}$ :

$$
E(V)=E_{\text {Int }}(V)+E_{\text {Image }}(V)+E_{E x t}(V)
$$

The image energy attracts the curve toward the features to be detected, such as edges or lines. The external energy $\left(E_{E x t}\right)$ can express additional contextual information. Thus, the curve is able to move dynamically from an initial position towards the desired boundary, without any further intervention. However, some problems still remain. Especially, the snake is sensitive to the initialization and it is unable to converge to the correct solution in the presence of high levels of noise.

Many improvements of the original formulation have been proposed in the literature. Here, we focus on methods dedicated to the detection of several lines or contours. The authors in $[2,3]$ present the simultaneous use of two snakes, which are connected in a one-to-one correspondence ("sandwich snake", "dual snakes"), both looking for the same contour. For closed curves in the "sandwich snake" approach, one snake $p$ is initially positioned inside the contour, and the other $q$ outside. The energy equation includes a new term related to a mutual attraction force (spring force), applied in the direction of the line connecting each pair of points $\left(p_{i}, q_{i}\right)$. For the dual snakes, both curves move towards a mean position, driven also by image forces and spring forces:

$$
\begin{aligned}
E_{E x t}(V) & =\int_{0}^{1} \frac{\lambda}{2}(V(s)-\operatorname{mean}(s))^{2} d s, \\
\operatorname{mean}(s) & =\frac{1}{2}(p(s)+q(s))
\end{aligned}
$$

where $V(s)=(x(s), y(s))$ is parameterized by arc length $s \in[0,1]$ and $\lambda$ controls the spring forces.

However, as in the original formulation, the external force applied on one point is only related to very local image information, the greylevel or the gradient at this point. To gain in robustness, it may be very interesting to integrate higher level information, related to the shape of the object, when this a priori information is available. Following this idea, the "dual snake" model [2] allows controlling the shape of the snake, by replacing the mean contour by a shape model in the energy term.

Parallelism is also an interesting feature that has been modelled in several works $[4,5,6]$. The "twin snake" algorithm [4] is an extension to the traditional snake, designed for the detection of two parallel contours simultaneously, in high-resolution images. Traditional snakes cannot detect accurately close edges, and the curve often jumps from one to the other. To overcome this problem, a mutual attraction force (spring force) is introduced, which is expressed at point $s$ as:

$$
E_{\text {ext }}(V(s))=\delta(s)\left(d(s)-d_{0}\right)^{2}
$$

where $\delta(s)$ is a weight factor, $d(s)$ the actual distance between the point $s$ and its twin partner and $d_{0}$ the desired distance. This latter parameter has to be initialized beforehand, which constitutes a strong limitation of this model. Moreover, this formulation assumes that the distance is constant all the way (strict parallelism), which is generally not the case in real images.

The "ribbon snake" [5] is modeled by a centerline $V(s)$ associated with a half width $w(s)$, defining the left and right borders of the ribbon, $V_{L}(s)$ and $V_{R}(s)$ respectively. It has been initially applied for automatic road extraction from aerial images. The image energy is defined at point $s$ as:

$$
E_{\text {Image }}(V(s))=\left(\nabla I\left(V_{L}(s)\right)-\nabla I\left(V_{R}(s)\right)\right) \cdot n(s)
$$


where $n(s)$ is the normal to the centerline and $\nabla I$ denotes the image gradient. This model does not allow explicitly controlling the variation of the ribbon width. Finally, the "twins ribbon" method [6] takes back the ideas developed in the "twin snake" and in the "sandwich snake" models. Four curves are evolving simultaneously: two of them inside the contour, the two others outside. The two outside contours are connected by pull forces to the corresponding inside contours (similarly to the sandwich model), while the inside contours are connected by push forces to each other (twin snake). This model is robust to high levels of noise and can locate boundaries under difficult conditions such as lack of gradient or occlusion. However, it requires a precise initialization, the prior knowledge of the ribbon width, and it is more computationally expensive than other models.

In the context of clinical studies related to ophthalmology, we need extracting thin, elongated and parallel structures that can be detected in several types of retina images, such as retinal layers in OCT images or retinal vessels in eye fundus images. These structures are difficult to segment since they present varying thicknesses and are generally weakly contrasted. Moreover, in some cases, diseases can affect the width or the grey levels of the searched structures.

In this paper, we propose a method to detect pairs of contours that are almost parallel. The advantages of our method are as follows:

(i) The distance between both contours has not to be known accurately as prerequisite. On the contrary, it is adjusted during the evolution process and can vary along the boundaries.

(ii) The parallelism constraint can be strengthened or relaxed locally by changing a weighting parameter.

(iii) All these interesting features are obtained without increasing the computational cost of the algorithm. Consequently, this model is well adapted to several applications, such as the ones mentioned above.

The remainder of the article is organized as follows: the novel Parallel Double Snake method is presented in Section 2, and experiments, including quantitative measurements, are discussed in Section 3.

\section{PARALLEL DOUBLE SNAKE MODEL}

Parallel Double Snakes are a new model for the simultaneous extraction of two almost parallel curves. The proposed model consists of a centerline, $V(s)=(x(s), y(s))$, parameterized by $s$ and a local half-thickness $b(s)$. It implicitly defines two parallel curves, $V_{1}$ and $V_{2}$.

Let us denote by $n(s)=\left(\begin{array}{c}n_{x}(s) \\ n_{y}(s)\end{array}\right)$ the normal vector to the curve $V(s)$ (Figure 1). Then, points of the curves $V_{1}$ and $V_{2}$ are given by:

$$
V_{1,2}(s)=V(s) \pm b(s) n(s)
$$

Our aim is to find the centerline $V(s)$ and the associated halfthickness $b(s)$, so that the curves $V_{1}$ and $V_{2}$ match the searched image contours, which are assumed to be almost parallel. For this, we introduce a new energy defined as:

$$
E(V, b)=E_{\text {Image }}\left(V_{1}\right)+E_{\text {Image }}\left(V_{2}\right)+E_{\text {Int }}(V)+R\left(V_{1}, V_{2}\right)
$$

where $E_{\text {Image }}$ and $E_{\text {Int }}$ are defined as in "classical" snakes, in order to attract the curves $V_{1}$ and $V_{2}$ towards the high intensity gradient values while preserving regularity properties of $V$ :

$$
\begin{gathered}
E_{\text {Image }}\left(V_{i}\right)=\int_{0}^{1} P\left(V_{i}(s)\right) d s=-\int_{0}^{1}\left|\nabla I\left(V_{i}(s)\right)\right|^{2} d s, \\
i=1,2
\end{gathered}
$$

$$
E_{\text {Int }}(V)=\frac{1}{2} \int \alpha(s)\left|\frac{\partial V(s, t)}{\partial s}\right|^{2}+\beta(s)\left|\frac{\partial^{2} V(s, t)}{\partial s^{2}}\right|^{2} d s
$$

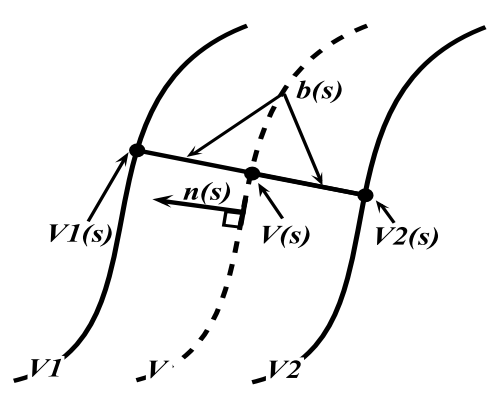

Fig. 1. Parametric representation of the Parallel Double Snakes.

In Equation 8, the weighting factors $\alpha(s)$ and $\beta(s)$ control the tension and rigidity of the centerline.

The last term $R\left(V_{1}, V_{2}\right)$ of Equation 6 involves the first derivative of $b(s)$. It controls the smoothness of the thickness variation and prevents it from sudden changes:

$$
R\left(V_{1}, V_{2}\right)=\int_{0}^{1} \varphi(s)\left(b^{\prime}(s)\right)^{2} d s
$$

For strictly parallel curves, $b(s)$ is constant and the related energy is zero. $\varphi(s)$ is a weighting parameter which may vary depending on $s$, to strengthen or relax the parallelism constraint. In the following, we assume that all weighting parameters are set to constant values. The minima of $E(V, b)$ are found by solving two coupled Euler-Lagrange equations. The first one expresses the minimization of $E(V, b)$ with respect to the centerline $V(s)$ :

$$
\left\{\begin{array}{l}
-\alpha x^{\prime \prime}(s)+\beta x^{\prime \prime \prime \prime}(s)+\frac{\partial P}{\partial x}\left(V_{1}(s)\right)+\frac{\partial P}{\partial x}\left(V_{2}(s)\right)=0 \\
-\alpha y^{\prime \prime}(s)+\beta y^{\prime \prime \prime \prime}(s)+\frac{\partial P}{\partial y}\left(V_{1}(s)\right)+\frac{\partial P}{\partial y}\left(V_{2}(s)\right)=0
\end{array}\right.
$$

Let us denote by $F(x, y)=-\nabla P(x, y)$ the image force at $(x, y)$. Equation 10 is very similar to the traditional formulation. The external force applied on each point of the centerline is defined as the sum of the forces applied on the corresponding border points $V_{1}(s)$ and $V_{2}(s)$. Thus, the curve moves globally towards the searched position.

The second Euler-Lagrange equation expresses the minimization of $E(V, b)$ with respect to the half-thickness $b(s)$ :

$$
\begin{aligned}
& n_{x} {\left[\frac{\partial P}{\partial x} V_{1}(s)-\frac{\partial P}{\partial x} V_{2}(s)\right] } \\
&+n_{y}\left[\frac{\partial P}{\partial y} V_{1}(s)-\frac{\partial P}{\partial y} V_{2}(s)\right]-2 \varphi b^{\prime \prime}(s)=0 \\
&\left\langle n, F\left(V_{2}(s)\right)-F\left(V_{1}(s)\right)\right\rangle-2 \varphi b^{\prime \prime}(s)=0
\end{aligned}
$$

This equation allows us to adjust the local distance between $V_{1}$ and $V_{2}$. Let us suppose that the centerline is at the correct position, but that $b(s)$ is locally too high (case 1 ) or too low (case 2). The local forces $F\left(V_{2}(s)\right)$ and $F\left(V_{1}(s)\right)$ point globally towards opposite directions (Figure 2 ), and the projection of their difference on the normal is negative (case 1) or positive (case 2). Thus, $b(s)$ increases or decreases so that $V_{1}$ and $V_{2}$ move toward the correct position. The last term of Equation 11, related to $b^{\prime \prime}(s)$, imposes the curves $V_{1}$ and 
$V_{2}$ to be regular.

To solve these equations, we classically introduce the time variable $t$ and discretize Equations 10 and 11, by using classical numerical approximations of derivatives (central difference in space, and backward difference in time).

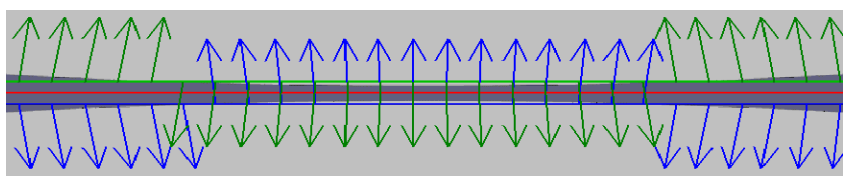

(a)

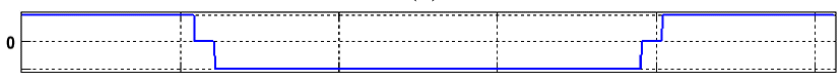

(b)

Fig. 2. Variations of the half-thickness $b(s)$ given by the second Euler-Lagrange Equation (11): (a) the obtained forces on each contour (green and blue arrows), (b) the variation of half-thickness.

The global minimization is realized by updating sequentially $V(s)$ and $b(s)$. Thus, the algorithm can be summarized as follows:

\author{
step1: Initialize the curve $V$ \\ Repeat \\ step 2: Calculate the normal vector $n$ \\ step 3: Update the curves $V_{1}$ and $V_{2}$ (Equation 5) \\ step 4: Deform the curve $V$ as a function of internal forces \\ and image forces (Equation 10) \\ step 5: Update the thickness parameter b (Equation 11) \\ until (Number of iterations)
}

Algorithm 1: Parallel Double Snake algorithm process.

The initialization of the curve $V$ and the parameter setting are specific for each type of application.

\section{EXPERIMENTS AND RESULTS}

In this section, we present two series of experiments, performed on two eye imaging modalities.

First, the Parallel Double Snake model has been applied on OCT images for the segmentation of some retinal layers. In our previous work [7], a complete segmentation method was proposed for healthy subjects. Unfortunately this method may fail in some pathological cases, where the destruction of photoreceptors induces a loss of definition of the inner nuclear layer (INL), and a loss of contrast of the hyper-reflective complex (HRC). Therefore, the INL and HRC are difficult to segment, especially the outer interface of each one. Here, we propose to first filter the original image using a nonlinear diffusion filter [8]. Then, the location of the inner side of the HRC (I. $\mathrm{S}$. HRC) and of the INL (I. S. INL) is obtained from the k-means algorithm, followed by a Markovian regularization [7] (respectively $k=4$ and $k=2$ classes). Indeed, the INL appears as the cluster corresponding to the lowest mean intensity. So, the inner side of the INL boundary can be easily deduced from the labeled image. A similar process is applied for the localization of the inner side of the
HRC. This provides, in both cases, an initialization of the centerline $V(s)$, chosen as a curve parallel to the interface found previously with an initial layer radius $b$, which is set to a constant value, adapted to each layer to be segmented. The proposed model is applied from this initialization, in order to get an accurate segmentation of the considered layers, as illustrated in Figure 3. The parameters are set to $b=30$ pixels, $\alpha=20, \beta=20, \varphi=20$ for the outer side of the HRC (O. S HRC), $b=10$ pixels, $\alpha=100, \beta=0, \varphi=20$ for the outer side of the INL (O. S. INL). In the same way, we can then apply our method to find the outer nuclear layer/inner segments of the photoreceptors (ONL/IS) interface, which is parallel to the inner side of the HRC previously detected. The parameters are set to $b=15$ pixels, $\alpha=50, \beta=10, \varphi=20$.

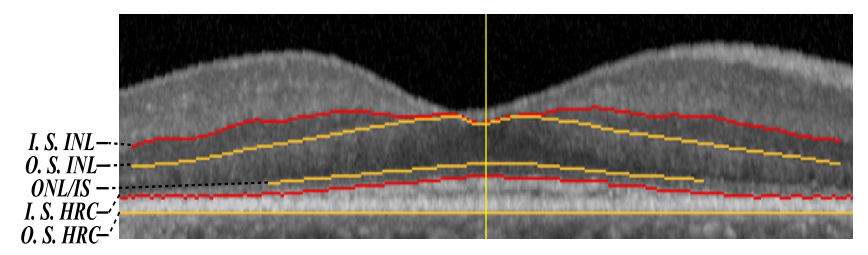

Fig. 3. Segmentation of some retinal layers in OCT images.

We compared the result of our method with the one provided by the "classical" snake algorithm in order to detect the HRC area. We used the same initial parameters, in both cases. As shown in Figure 4, we can see that the proposed method successfully segments the HRC zone (Figure 4(b)), while the "classical" snake method fails in the region outside fovea (Figure 4(a)). The parallelism constraint prevents the snake to escape towards high gradient present in the choroid (under the HRC).

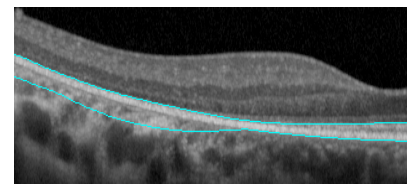

(a)

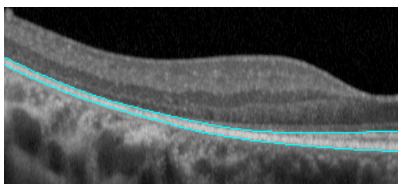

(b)
Fig. 4. A comparison between (a) "classical" snake model and (b) the Parallel Double Snake model.

OCT imaging was performed on 25 images, from 8 retinitis pigmentosa subjects, with various grades of pathology evolution. A vertical and a horizontal cross-section were generally acquired for each eye. This study was conducted in accordance with French ethics regulation and all subjects gave informed consent to participate. The same parameters were used for all images. The results were evaluated by comparing the obtained segmentations with manual segmentations performed by a highly experimented medical doctor, on 5 OCT images. Intra-expert variability was assessed too. The quantitative results are summarized in Table 1, showing a very good agreement between automated and manual results (and within the range of intra-expert variability).

As shown in Table 1, the intra-expert errors are on average of the same order of magnitude as the automatic/manual errors. Moreover, for the outer side of the HRC and the ONL/IS interface, the MSE between our method and the manual segmentation is lower than the intra-expert variability.

The index of similarity $(S)$ was also computed for all regions. This index is sensitive to variations in shape, size and position and a value of $S>0.7$ indicates a strong agreement [9]. We found that $S$ is in all 
cases greater than 0.7 and that it ranges from 0.81 for the IS layer to 0.91 for the HRC layer. These evaluations confirm that the Parallel Double Snake model allows us to get accurate and robust segmentations, which are comparable to the expert's ones.

\begin{tabular}{|l|c|c|}
\hline Layer & Automatic vs. manual & Intra-expert variability \\
\hline O. S. HRC & $1.06 \pm 0.22$ & $1.28 \pm 0.19$ \\
ONL/IS & $1.23 \pm 0.44$ & $1.30 \pm 0.18$ \\
O. S. INL & $1.35 \pm 0.51$ & $1.14 \pm 0.29$ \\
\hline
\end{tabular}

Table 1. Mean square error (MSE) between manual and automated segmentations (in pixels).

In the second experiment, the proposed model has been applied on eye fundus images for the segmentation of retinal vessels in the vicinity of the optical disk. The vessels are enhanced, based on morphological operations and linear filtering, and segmented. A graph is then extracted from the skeleton of the binary image, providing a high-level representation of the vascular tree [10]. A region of interest is then defined as the ring delimited by the circles of radius $2 R$ and $3 R$ (Figure 5), where $R$ is the optical disk radius. The proposed method is then used to refine the segmentation of the branches in this region. The centerline $V(s)$ is initialized as the skeleton branch. The vessel radius $b$ is estimated by computing the Euclidean distance transform to the skeleton branch and retaining the maximum value found on the vessel borders. The Parallel Double Snake model is run from this initialization to get an accurate location of the vessel boundaries, as illustrated in Figure 5. The parameters are set to $\alpha=20, \beta=0, \varphi=50$.

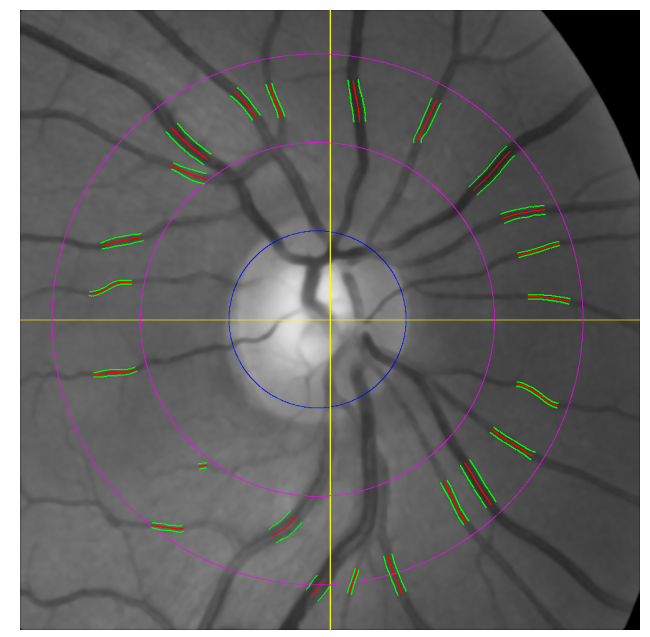

Fig. 5. Segmentation of retina vessels near the optical disk in eye fundus images.

The method was applied on a database of 30 images acquired in color or grey levels. Compared to the initial segmentation [10], the vessels are much more precisely delineated, whatever their thicknesses and the image contrast. These results, visually assessed by the expert, show again the robustness and the accuracy of the Double Parallel Snake method.

From the obtained segmentation results, measurements of arteria and vein diameters, and their combination into central arteriolar and venular equivalents (A/V ratio) can be then derived. From a clinical point of view, such measures are used for the follow up of various retinal vascular abnormalities, such as arteriolar narrowing, or arteriovenous $(\mathrm{A} / \mathrm{V})$ crossing changes.

\section{CONCLUSION}

In this paper, a new parametric deformable model has been proposed. It incorporates a priori information by adding a parallelism constraint compared to other models. The Parallel Double Snake algorithm reduces the complexity of evolution as well as the number of parameters, and it is well adapted to several applications. The quantitative evaluation shows a very good agreement and high correlation between the manual and the automated segmentations. Such segmentation results will help ophthalmologists to improve the accuracy of diagnosis of blinding retinal diseases. A more detailed evaluation will be performed in future work to assess the effectiveness of the proposed method compared to well known methods.

Acknowledgments: This work was partially funded by a CIFRE grant from Fovea Pharmaceuticals and the French Ministry of Research (Contract CIFRE 897-2008).

\section{REFERENCES}

[1] M. Kass, A. Witkin, and D. Terzopoulos, "Snakes: Active contour models," International Journal of Computer Vision, vol. 1, no. 4, pp. 321-331, 1988.

[2] S. R. Gunn and M. S. Nixon, "A robust snake implementation; a dual active contour," IEEE Transactions on Pattern Analysis and Machine Intelligence, vol. 19, pp. 63-68, 1997.

[3] F. A. Velasco and J. L. Marroquín, "Robust parametric active contours: the sandwich snakes," Machine Vision and Application, vol. 12, pp. 238-242, 2001.

[4] M. Kerschner, "Twin snakes for determining seam lines in orthoimage mosaicking," International Archives of Photogrammetry and Remote Sensing, vol. 33, pp. 454-461, 2000.

[5] I. Laptev, H. Mayer, T. Lindeberg, W. Eckstein, C. Steger, and A. Baumgartner, "Automatic extraction of roads from aerial images based on scale space and snakes," Machine Vision and Application, vol. 12, pp. 23-31, 2000.

[6] B. Al-Diri, A. Hunter, and D. Steel, "An active contour model for segmenting and measuring retinal vessels," IEEE Transactions on Medical Imaging, vol. 28, no. 9, pp. 1488-1497, 2009.

[7] I. Ghorbel, F. Rossant, I. Bloch, S. Tick, and M. Paques, "Automated Segmentation of Macular Layers in OCT Images and Quantitative Evaluation of Performances," Pattern Recognition, vol. 44, no. 8, pp. 1590-1603, 2011.

[8] J. Weickert, B.M.Ten Haar Romeny, and M.A. Viergever, "Efficient and reliable schemes for nonlinear diffusion filtering," IEEE Transactions on Image Processing, vol. 7, no. 3, pp. 398410, 1998.

[9] A. Zijdenbos, B. Dawant, R. Margolin, and A. Palmer, "Morphometric Analysis of White Matter Lesions in MR Images: Method and Validation," IEEE Transactions on Medical Imaging, vol. 13, no. 4, pp. 716-724, 1994.

[10] F. Rossant, M. Badellino, A. Chavillon, I. Bloch, and M. Paques, "A morphological approach for vessel segmentation in eye fundus images, with quantitative evaluation," Journal of Medical Imaging and Health Informatics, vol. 1, no. 1, pp. 42-49, Mar. 2011. 